\title{
PROPOSING A NEW MODEL ON DATA ENVELOPMENT ANALYSIS BY CONSIDERING NON DISCRETIONARY FACTORS AND A REVIEW ON PREVIOUS MODELS
}

\author{
G.R. Jahanshahloo ${ }^{1}$, F. Hosseinzadeh Lotfi ${ }^{1}$, N. Shoja ${ }^{2}$, A.Gholam Abri ${ }^{*}$, \\ M. Fallah Jelodar ${ }^{1}$, Kamran Jamali firouzabadi ${ }^{2}$ \\ ${ }^{1}$ Department of Mathematics, Science and Research Branch,Islamic Azad \\ University, Tehran, Iran. \\ ${ }^{2}$ Department of Mathematics, Firoozkooh branch, Islamic Azad University, Firoozkooh, \\ Iran. \\ Amirgholamabri@gmail.com
}

\begin{abstract}
Discretionary models of data envelopment analysis (DEA) assume that all inputs and outputs are discretionary, i.e., controlled by the management of each decision making unit (DMU) and varied at its discretion. In any realistic situation, however, there may exist exogenously fixed or non-discretionary inputs or outputs that are beyond the control of a DMU's management. There are some models that incorporate non-discretionary inputs into DEA models. This paper reviews these approaches, providing a discussion of strengths and weaknesses and highlighting potential limitations. Moreover, a new method is developed that overcomes existing weaknesses.
\end{abstract}

Key Words- Data Envelopment Analysis,Non-Discretionary, Efficiency, Exogenously

\section{INTRODUCTION}

Data envelopment analysis (DEA) which introduced by Charnes et al. [4] (CCR) and extended by Banker et al. [1] (BCC), is a useful method to evaluate relative efficiency of multiple-inputs and multiple-outputs units based on observed data.

On the basis of various production process assumptions, a number of different models has been developed.

Standard DEA assumes that the assessed units (DMUs) are homogeneous, i.e. they perform the same tasks with similar objectives, consume similar inputs and produce similar outputs, and operate in similar operational environments. Often the assumption of homogeneous environments is violated and factors that describe the differences in the environments need to be included in the analysis. These factors, and others outside the control of the DMUs, are frequently called non-discretionary factors. There are some approaches which seems to be more general. These approaches are developed for controlling the non-discretionary inputs. The DEA model is coined by Banker and Morey [2] for fulfilling what above said. Convexity is an assumption by considering either discretionary or non-discretionary inputs. These classes of inputs were treated differently, however, by not allowing radial reduction in the nondiscretionary inputs.

Ruggiero [11] extended this model by dropping the convexity constraint associated with the non-discretionary inputs. Rather, non-discretionary inputs were treated as shift factors leading to multiple frontiers and restrictions were placed on the weights to 
exclude DMUs with more favorable levels of the non-discretionary factor.

The approach that is considered here as a third one is introduced by Ray [9], which does not consider the non-discretionary inputs in the DEA model in the first stage. The non-discretionary inputs are controlled in the second stage of regression, which permits an adjusted measure of technical efficiency to enter the model. A hybrid model is announced by Ruggiero [13] that have three stages for allowing the multiple nondiscretionary inputs to be paid attention. Simulation analysis (Ruggiero [13]) showed that multiple stage models of Ray and Ruggiero have a superior level in comparison with Banker and Morey model, and are acted better.

In order to compare Banker and Morey model with the stochastic frontier model with one exogenous variable, a simulation analysis is used by $\mathrm{Yu}$ [14]. The cross-sectional stochastic frontier approach has been depicted by Ondrich and Ruggiero [8] to be of limited value since it does not really allow measurement error. Other concluded result by $\mathrm{Yu}$ are consistent with Ruggiero [11].

Moreover, a revised model will be proposed that will produce an undistorted efficiency measure by Ruggiero [12]. As discussed in that paper and illustrated with simulation analysis, the performance of the existing model declines as the relationship between non-discretionary inputs and true but unobserved efficiency gets stronger. In addition to discussing the problem, that paper introduced a new DEA model which overcomes the identified problems. One shortcoming, however, was the reliance on parametric techniques to identify this relationship.

The purpose of this paper is to compare the three approaches, highlighting potential strengths and weaknesses. A new model is developed that overcomes identified weaknesses.

The current paper proceeds as follows. section 2 discusses the basic DEA models and develops a methodology for treating non-discretionary and section 3 provides a practical example. Finally, conclusions are given.

\section{PRELIMINARIES AND DEVLOPMENT OF THE DEA MODELS IN THE PRESENCE OF NON-DISCRETIONARY INPUTS}

Data Envelopment Analysis (DEA) is a technique that has been used widely in the supply chain management literature. This non-parametric, multi-factor approach enhances our ability to capture the multi-dimensionality of performance discussed earlier. More formally, DEA is a mathematical programming technique for measuring the relative efficiency of decision making units (DMUs) where each DMU has a set of inputs used to produce a set of outputs.

Consider $D M U_{j},(j=1, \ldots, n)$, where each DMU consumes $\mathrm{m}$ inputs to produce $\mathrm{s}$ outputs. Suppose the observed input and output vectors of $D M U_{j}$ be $X_{j}=\left(x_{1 j}, \ldots, x_{m j}\right)$ and $Y_{j}=\left(y_{1 j}, \ldots, y_{s j}\right)$ respectively, and let $X_{j} \geq 0$ and $X_{j} \neq 0$ and $Y_{j} \geq 0$ and $Y_{j} \neq 0$.

In DEA, the CCR model is one of the most important radial models, evaluates the relative efficiency of a specific $D M U_{o}, o \in(1, \ldots, n)$, with respect to a set of CCRfrontier DMUs.

We can write an input-oriented CCR model as follows: 


$$
\begin{aligned}
& \text { Min } \theta \\
& \text { s.t. } \sum_{j=1}^{n} \lambda_{j} x_{i j} \leq \theta x_{i o} \quad, i=1, \ldots, m \\
& \sum_{j=1}^{n} \lambda_{j} y_{r j} \geq y_{r o} \quad, r=1, \ldots, s \\
& \lambda_{j} \geq 0 \quad, j=1, \ldots, n
\end{aligned}
$$

Moreover an input-oriented $\mathrm{BCC}$ model is as follows:

$$
\begin{aligned}
& \operatorname{Min} \theta \\
& \text { S.t } \quad \sum_{j=1}^{n} \lambda_{j} x_{i j} \leq \theta x_{i o}, \quad i=1, \ldots, m \\
& \sum_{j=1}^{n} \lambda_{j} y_{r j} \geq y_{r o}, \quad r=1, \ldots, s \\
& \sum_{j=1}^{n} \lambda_{j}=1, \\
& \lambda_{j} \geq 0, \quad j=1, \ldots, n
\end{aligned}
$$

One major problem with a radial measure of technical efficiency is that, it does not reflect all identifiable potential for increasing outputs and reducing inputs. In economics, the concept of efficiency is intimately related to the idea of pareto optimality. An input-output bundle is not pareto optimal if there remains the possibility of any net increase in outputs or net reduction in inputs. When positive output and input slacks are present at the optimal solution of a CCR LP problem, the corresponding radial projection of an observed input-output combination does not meet the criterion of pareto optimality and should not be qualified as an efficient point.

A non-radial Pareto-Koopmans measure of technical efficiency of the input-output pair $\left(X_{o}, Y_{o}\right)$ can be computed as:

$$
\begin{array}{cl}
\text { Min } \quad \Gamma=\frac{\frac{1}{m} \sum_{i=1}^{m} \theta_{i}}{\frac{1}{S} \sum_{r=1}^{s} \phi_{r}} & \\
\text { s.t. } \sum_{j=1}^{n} \lambda_{j} x_{i j} \leq \theta_{i} x_{i o} & , i=1, \ldots, m \\
\sum_{j=1}^{n} \lambda_{j} y_{r j} \geq \phi_{r} y_{r o} & , r=1, \ldots, s \\
\lambda_{j} \geq 0 & , j=1, \ldots, n \\
\theta_{i} \leq 1 & , i=1, \ldots, m \\
\phi_{r} \geq 1 & , r=1, \ldots, s
\end{array}
$$


In this model $\left(X_{o}, Y_{o}\right)$ is Pareto-Koopmans efficient if and only if $\phi_{r}^{*}=1$ for each output $\mathrm{r}$ and $\theta_{i}^{*}=1$ for each input $\mathrm{i}$ implying $\Gamma=1$.

The objective function in this mathematical programming problem is nonlinear. But it is possible to linearize it as [10].

Up to this point, we have assumed that all inputs and outputs can be varied at the discretion of managementore other users. These may be called " discretionary variables."

"Non-discretionary variables," not subject to management control, may also need to be considered.

Assume that there are $\mathrm{n}$ DMUs, where each $D M U_{j}(j=1, \ldots, n)$, uses $\mathrm{t}$ different discretionary inputs, $x_{i j}(i=1, \ldots, t)$, and $\mathrm{k}$ different non-discretionary inputs, $z_{i j}(i=1, \ldots, k)$, where $t+k=m$, to produce s different outputs $y_{r j}(r=1, \ldots, s)$.

There are some models that incorporate non-discretionary inputs into DEA models. Banker and Morey provided the first model by modifying the constraints on the fixed factors within the DEA model. This model differs from the original DEA model by breaking the link between non-discretionary inputs and efficiency.

This model is as follows:

$$
\begin{aligned}
& \operatorname{Min} \quad \theta \\
& \text { s.t. } \sum_{j=1}^{n} \lambda_{j} x_{i j} \leq \theta x_{i o} \quad, i=1, \ldots, t \\
& \sum_{j=1}^{n} \lambda_{j} z_{i j} \leq z_{i o} \quad, i=1, \ldots, k \\
& \sum_{j=1}^{n} \lambda_{j} y_{r j} \geq y_{r o} \quad, r=1, \ldots, s \\
& \sum_{j=1}^{n} \lambda_{j}=1 \\
& \lambda_{j} \geq 0 \quad, j=1, \ldots, n
\end{aligned}
$$

There is a great similarity between fixed factor constraints and constraints on the discretionary inputs; both are modified, however, to break the link between efficiency and the fixed factors, the fixed factors of production are being under control by the modification. The recent subject is done by requiring a convex combination of the referent production possibilities in order to gain an environment which is not better than the DMU that is going to be analyzed.

It is showed, by Ruggiero [11] that the referent "production possibility" may not be feasible. This results, because of returns to scale, should be defined relative only to discretionary inputs. Emphasis on convexity with consideration the non-discretionary inputs concludes to improper restriction of the production possibility sets and distorted efficiency measurement.

Then several authors have proposed methods for analyzing performance in the presence of non-discretionary inputs. One of the model have proposed by Ruggiero [11] 
is as follows :

$$
\begin{aligned}
& \operatorname{Min} \theta \\
& \text { S.t } \sum_{j=1}^{n} \lambda_{j} x_{i j} \leq \theta x_{i o} \quad, i=1, \ldots, t \\
& \sum_{j=1}^{n} \lambda_{j} y_{r j} \geq y_{\text {ro }} \quad, r=1, \ldots, s \\
& \lambda_{j}=0, \quad \text { if } \exists z_{i j}>z_{i o}, i=1, \ldots, k \\
& \sum_{j=1}^{n} \lambda_{j}=1 \\
& \lambda_{j} \geq 0 \quad, j=1, \ldots, n
\end{aligned}
$$

The model explicitly explains some limitations for the comparison set to exclude DMUs which encounter a more pleasant and better environment. Like the model (4), this model needs to have a priority specification of the continuous non-discretionary variables. By increasing the number of fixed factors continuously, the likelihood of identifying a DMU, as efficient by default, increases. This does not consider the comparisons between a given DMU and another DMU that as a whole, has the same or not better environment, even though it has a more favorable level of at least one nondiscretionary input. The matter makes us a suggestion an inherent weakness of the Ruggiero model.

To remove these weaknesses, Ray [9] developed an alternative two-stage. In the first stage the BCC model is employed using only the discretionary inputs.

Ray [9] showed that regression analysis can be used in the second stage to factor out the effect that the environment has on production. In his study, the first-stage index FS was regressed on the fixed factors of production. Ignoring observation subscripts, the second-stage regression model is specified as

$$
F S=\alpha+\beta_{1} z_{1}+\ldots+\beta_{k} z_{k}+\varepsilon
$$

Note that if the positive change $d z_{i}$ in $z_{i}$ represents a more favorable environment, then $\beta_{i}>0$. Based on the regression results, Ray's measure RAY of technical efficiency is computed as

$$
R A Y=F S-\alpha-\beta_{1} z_{1}-\ldots-\beta_{k} z_{k} \text {. }
$$

Due to the nature of regression, this index will have mean 0 . The index, however, can be transformed by adjusting the intercept $\alpha$ so that RAY is non-negative. The distortions introduced in the original DEA model from the exclusion of the environmental variables is factored out via regression analysis [13].

The first advantage of the two-stage approach is that it can be computed. The twostage approach requires solving the original DEA model once. This offers considerable flexibility and permits sensitivity analysis in the second stage. So, various sets of nondiscretionary inputs can be examined. The added flexibility overcomes weaknesses identified in Ruggiero's public sector DEA model as well. If numerous variables of nondiscretionary exist, the regression analysis provides the means to implicitly weigh the contribution each variable has on the first-stage estimate. 
The two-stage approach has also some potential weaknesses. The second stage of regression needs a priori circumstances of functional form. If the functional form is misspecified, then Ray's measure will be distorted. In this way, Ray's model encounters problems like regression based approaches. Moreover, it is possible that inefficiency will be overstated, because of adjusting are made based on the two-sided error.

As showed above, the Ruggiero model is not able completely to weigh the importance of each non-discretionary variable in production. Therefore, with increasing the number of non-discretionary inputs ceteris paribus, the Ruggiero model will be more likely to overstate efficiency. The importance of theses non-discretionary inputs, however, is revealed in the second-stage regression of Ray's model. Given the parameters, it is possible to obtain an overall index $\mathrm{Z}$ of environmental harshness as $Z=\sum_{i=1}^{k} \beta_{i} z_{i}$

Given this index of environmental harshness, the Ruggiero input-oriented (variable returns to scale) efficiency measure for $D M U_{o}$ can be specified as a three-stage procedure. First, the BCC model is solved and the second-stage regression (*) is performed. After construction of $\mathrm{Z}$, the following third-stage linear program is solved:

$$
\begin{aligned}
& \text { Min } \theta \\
& \text { s.t. } \sum_{j=1}^{n} \lambda_{j} x_{i j} \leq \theta x_{i o} \quad, i=1, \ldots, t \\
& \sum_{j=1}^{n} \lambda_{j} y_{r j} \geq y_{r o} \quad, r=1, \ldots, s \\
& \sum_{j=1}^{n} \lambda_{j}=1 \\
& \lambda_{j}=0 \text { if } Z_{j}>Z_{o} \\
& \lambda_{j} \geq 0 \quad, j=1, \ldots, n
\end{aligned}
$$

There are a few advantages to using this new model. At first, suppose that the second-stage regression introduces unbiased estimates of the parameter weights, the model gains the desirable properties of the Ruggiero model. Second, the model masters the defined weakness of identifying DMUs as efficient by default innate in the Ruggiero model. Instead, this model is capable for weighting the importance of the nondiscretionary inputs. Ray's model uses also the error term for measuring the efficiency and consequently, it will be sensitive to mis-specification.

This model only uses the parameter weights to construct the environmental harshness index. As a result, distributional assumptions are not made regarding efficiency. Further, this new model (unlike Ray's models) maintains other desirable properties of the original DEA models. In particular, this model controls for nondiscretionary factors and uncovers the efficient referent set. As a result, the nature of scale economies can be revealed. Finally, potentially useful information regarding causes of inefficiency is not lost since inefficient DMUs can be compared to its efficient referent set [13].

One key assumption in model (6) is that true efficiency is not correlated with nondiscretionary factor. To overcome these difficulties, Ruggiero modified the above model in following: 


$$
\begin{aligned}
& \text { Min } \theta \\
& \text { s.t. } \sum_{j=1}^{n} \lambda_{j} x_{i j} \leq \theta x_{i o} \quad, i=1, \ldots, t \\
& \sum_{j=1}^{n} \lambda_{j} y_{r j} \geq y_{r o} \quad, r=1, \ldots, s \\
& \sum_{j=1}^{n} \lambda_{j}=1 \\
& \lambda_{j}=0 \text { if } Z_{j}>Z_{o}+\delta(z), \delta(z)>0 \\
& \lambda_{j} \geq 0 \quad, j=1, \ldots, n
\end{aligned}
$$

Model (7) differs from model (6) by relaxing the constraint that restricts DMUs with a higher level of the non-discretionary input from the reference group.

Now, DMUs with higher levels of the non-discretionary input can be included as long as the difference between non-discretionary levels is not greater than $\delta(z)$. By relaxing this constraint, DMUs with a more favorable environment can be included in the referent set, which essentially controls for the correlation between efficiency and the non-discretionary environment, [12].

Unfortunately, the revised model requires an additional assumption on the production technology and parametric specification in a second stage. Moreover, as pointed above, this approach theoretically hasn't any difficulty, but applying it practically and obtaining $\delta(z)$ will not be simply performed.

In order to overcome any difficulties, we will develop a method that simply will be utilized in applied aspects. In addition to solve the former problems, decision making units will acquired a real efficiencies in presence of non-discretionary inputs.

For this purpose, first, we will rewrite model (3) in presence of non-discretionary inputs as follows and we find $\delta_{1 o}^{*}, \ldots, \delta_{k o}^{*}$.

$$
\begin{array}{cl}
\operatorname{Min} \quad \Gamma=\frac{\frac{1}{m}\left(\sum_{i=1}^{t} \theta_{i}+\sum_{i=1}^{k} \delta_{i}\right)}{\frac{1}{s} \sum_{r=1}^{s} \phi_{r}} \\
\text { s.t. } \sum_{j=1}^{n} \lambda_{j} x_{i j} \leq \theta_{i} x_{i o} \quad, i=1, \ldots, t \\
\sum_{j=1}^{n} \lambda_{j} z_{i j} \leq \delta_{i} z_{i o} & , i=1, \ldots, k \\
\sum_{j=1}^{n} \lambda_{j} y_{r j} \geq \phi_{r} y_{r o} & , r=1, \ldots, s \\
\lambda_{j} \geq 0 & , j=1, \ldots, n \\
\theta_{i} \leq 1 & , i=1, \ldots, t \\
\delta_{i} \leq 1 & , i=1, \ldots, k \\
\phi_{r} \geq 1 & , r=1, \ldots, s
\end{array}
$$


In continue, we apply the following model :

$$
\begin{array}{rr}
\text { Min } \quad \Gamma=\frac{\frac{1}{t} \sum_{i=1}^{t} \theta_{i}}{\frac{1}{s} \sum_{r=1}^{s} \phi_{r}} & \\
\text { s.t. } \sum_{j=1}^{n} \lambda_{j} x_{i j} \leq \theta_{i} x_{i o} & , i=1, \ldots, t \\
\sum_{j=1}^{n} \lambda_{j} y_{r j} \geq \phi_{r} y_{r o} & , r=1, \ldots, s \\
\lambda_{j}=0 \text { if } \sum_{i=1}^{k} \delta_{i}^{*} z_{i o}<\sum_{i=1}^{k} \delta_{i}^{*} z_{i j} & , j=1, \ldots, n \\
\lambda_{j} \geq 0 & , j=1, \ldots, n \\
\theta_{i} \leq 1 & , i=1, \ldots, t \\
\phi_{r} \geq 1 & , r=1, \ldots, s
\end{array}
$$

Follow to the last model, we obtain true efficiency of evaluated DMUs.

Using the proposed method, some advantages would be appeared. This method overcomes the identified weakness of identifying DMUs as efficient by default inherent in the Ruggiero models. Importantly, as the number of non-discretionary factors increases, this method can compare all non-discretionary factors, so this method obtain true efficiency. Specially, the proposed method can be utilized simply in practical examples.

\section{A PRACTICAL EXAMPLE}

In this section, we work out an applied example to illustrate the proposed model. So consider 30 bank branches in Iran with 3 discretionary inputs and 2 non-discretionary inputs and 4 outputs. First we apply model (8) for each $D M U_{j},(j=1, \ldots, n)$ and we find $\delta_{i j}^{*},(i \in N . D)$.

In what follows, we apply model (9) and obtain true efficiency of evaluated DMUs. Data and results are summarized in table 1: 
Table 1. Data and Result

\begin{tabular}{|c|c|c|c|c|c|c|c|c|c|c|c|}
\hline \multirow[t]{2}{*}{ DMUs } & \multicolumn{3}{|c|}{ Discretionary } & \multicolumn{2}{|c|}{$\begin{array}{c}\text { Non- } \\
\text { Discretionary }\end{array}$} & \multicolumn{4}{|c|}{ Output } & \multirow[t]{2}{*}{ Ruggiero } & \multirow[t]{2}{*}{$\begin{array}{c}\text { Proposed } \\
\text { Model }\end{array}$} \\
\hline & I1 & $\mathrm{I} 2$ & $\mathrm{I} 3$ & $\mathrm{Z} 1$ & $\mathrm{Z} 2$ & $\mathrm{O} 1$ & $\mathrm{O} 2$ & $\mathrm{O} 3$ & $\mathrm{O} 4$ & & \\
\hline 1 & 0.93 & 0.44 & 0.18 & 0.70 & 0.82 & 0.05 & 0.10 & 0.00 & 0.58 & 0.56 & 1.00 \\
\hline 2 & 0.86 & 0.37 & 0.15 & 0.96 & 0.68 & 0.05 & 0.06 & 0.01 & 0.68 & 0.73 & 1.00 \\
\hline 3 & 1.00 & 0.33 & 0.10 & 0.88 & 0.79 & 0.08 & 0.13 & 0.00 & 0.79 & 0.69 & 1.00 \\
\hline 4 & 1.00 & 0.20 & 0.34 & 0.43 & 1.00 & 0.22 & 0.16 & 0.00 & 1.00 & 1.00 & 1.00 \\
\hline 5 & 0.93 & 0.36 & 0.19 & 0.79 & 0.78 & 0.09 & 0.08 & 0.01 & 0.79 & 0.76 & 1.00 \\
\hline 6 & 0.93 & 0.44 & 0.00 & 1.00 & 0.78 & 0.06 & 0.03 & 0.00 & 0.51 & 1.00 & 0.66 \\
\hline 7 & 0.73 & 0.26 & 0.00 & 0.53 & 0.62 & 0.04 & 0.08 & 0.00 & 0.54 & 1.00 & 0.66 \\
\hline 8 & 0.67 & 0.11 & 0.00 & 0.73 & 0.57 & 0.02 & 0.05 & 0.00 & 0.32 & 0.83 & 1.00 \\
\hline 9 & 0.67 & 0.25 & 0.36 & 0.85 & 0.50 & 0.06 & 0.10 & 0.00 & 0.63 & 0.83 & 0.61 \\
\hline 10 & 0.53 & 0.17 & 0.00 & 0.34 & 0.50 & 0.07 & 0.14 & 0.01 & 0.40 & 1.00 & 0.66 \\
\hline 11 & 0.80 & 0.26 & 0.03 & 0.64 & 0.69 & 0.06 & 0.05 & 0.00 & 0.80 & 0.85 & 0.58 \\
\hline 12 & 0.60 & 0.05 & 0.00 & 0.62 & 0.50 & 0.07 & 0.07 & 0.00 & 0.48 & 1.00 & 1.00 \\
\hline 13 & 0.60 & 0.06 & 0.02 & 0.61 & 0.51 & 0.07 & 0.04 & 0.00 & 0.36 & 0.91 & 1.00 \\
\hline 14 & 0.73 & 0.12 & 0.13 & 0.63 & 0.62 & 0.05 & 0.07 & 0.00 & 0.44 & 0.63 & 1.00 \\
\hline 15 & 0.67 & 0.06 & 0.00 & 0.77 & 0.54 & 0.05 & 0.06 & 0.01 & 0.56 & 1.00 & 1.00 \\
\hline 16 & 0.60 & 0.14 & 0.10 & 0.48 & 0.53 & 0.03 & 0.05 & 0.00 & 0.43 & 0.77 & 1.00 \\
\hline 17 & 0.80 & 0.18 & 0.00 & 0.47 & 0.76 & 0.40 & 0.12 & 0.00 & 0.94 & 1.00 & 1.00 \\
\hline 18 & 0.40 & 0.08 & 0.01 & 0.25 & 0.37 & 0.03 & 0.01 & 0.00 & 0.42 & 1.00 & 1.00 \\
\hline 19 & 0.93 & 0.34 & 0.50 & 0.62 & 0.84 & 0.14 & 0.32 & 0.02 & 0.64 & 1.00 & 1.00 \\
\hline 20 & 0.67 & 0.17 & 0.00 & 0.38 & 0.63 & 0.09 & 0.23 & 0.00 & 0.74 & 1.00 & 1.00 \\
\hline 21 & 0.73 & 0.16 & 0.00 & 0.58 & 0.65 & 0.17 & 0.32 & 0.01 & 0.60 & 1.00 & 1.00 \\
\hline 22 & 0.40 & 0.10 & 0.00 & 0.31 & 0.33 & 0.03 & 0.02 & 0.00 & 0.33 & 1.00 & 1.00 \\
\hline 23 & 0.80 & 0.14 & 0.00 & 0.49 & 0.79 & 0.16 & 0.28 & 0.05 & 0.65 & 1.00 & 1.00 \\
\hline 24 & 0.40 & 0.11 & 0.00 & 0.37 & 0.32 & 0.02 & 0.04 & 0.00 & 0.23 & 1.00 & 0.66 \\
\hline 25 & 0.47 & 0.17 & 0.01 & 0.22 & 0.48 & 0.03 & 0.02 & 0.00 & 0.27 & 1.00 & 1.00 \\
\hline 26 & 0.80 & 0.25 & 0.19 & 0.49 & 0.74 & 0.06 & 0.03 & 0.00 & 0.82 & 0.86 & 0.55 \\
\hline 27 & 0.80 & 0.16 & 0.43 & 0.67 & 0.69 & 0.08 & 0.14 & 0.00 & 0.64 & 0.69 & 1.00 \\
\hline 28 & 0.46 & 0.19 & 0.10 & 0.37 & 0.41 & 0.03 & 0.04 & 0.00 & 0.27 & 1.00 & 1.00 \\
\hline 29 & 0.73 & 0.11 & 0.00 & 0.42 & 0.69 & 0.68 & 0.27 & 0.00 & 0.87 & 1.00 & 1.00 \\
\hline 30 & 0.26 & 0.07 & 0.10 & 0.18 & 0.26 & 0.03 & 0.02 & 0.00 & 0.24 & 1.00 & 1.00 \\
\hline
\end{tabular}

\section{CONCLUDING REMARKS}

In any realistic situation, however, there may exist exogenously fixed or nondiscretionary inputs or outputs that are beyond the control of a DMU' $s$ management.

This paper has focused on the presence of non-discretionary inputs in production processes and in the programming models used to measure inefficiency.

Five existing models were discussed and their strengths and weaknesses were identified. Moreover, this paper introduced a new DEA model that overcomes the identified problems.

There are some advantages by using this proposed method. This method overcomes the identified weakness of identifying DMUs as efficient by default inherent in the models which have been proposed. Moreover, as the number of non-discretionary factors increases, this method can compare all non-discretionary factors, so this method obtain true efficiency. Specially, the proposed method can be utilized simply in practical examples. 


\section{REFERENCES}

1. Banker, R.D., Charnes, A., Cooper, W.W., Some models for estimating technical and scale inefficiencies in data envelopment analysis. Management Science 30 (9), 10781092, 1984.

2. Banker, R.D, Morey, R.C, Efficiency analysis for exogenously fixed inputs and outputs. European Journal of Operational Research 553-565, 1986.

3. Bradford, D., Malt, R., Oates, W., The rising cost of local public services.Some evidences and rreflections, National tax Journal 22 185-202, 1969.

4. Charnes, A., Cooper, W.W., Rhodes, E., Measuring the efficiency of decision making units. European Journal of Operational Research 2, 429-444, 1978.

5. Fare, R., Grosskof, S., Lovell, C.A.K., Production frontiers, Cambridge University Press, New York, 1994.

6. Fare, R., Grosskof, S., Lovell, C.A.K., The management of efficiency of production. Klower-Nijhoff, Boston, MA, 1985.

7. Farell, M.J, The measurement of productive efficiency. Journal of the royal statistical science series A, General 120, 253-281, 1957.

8. Ondrich,J;Ruggiero,J, Efficiency measurement in the Stochastic frontier model. European Journal of Operational Research 129, 434-442, 2001.

9. Ray, S.c., Resource use eficiency in public schools. A study of connecticut data, Management Science 37, 1620-1628, 1991.

10. Ray, Subhash.c., Data envelopment analysis: theory and techniques for economics and operations research. Published by the press syndicate of the university of Cambridge, 2004.

11. Ruggiero, J., On the measurement of technical efficiency in the public sector, European Journal of Operational Research553-565, 1996.

12. Ruggiero, J., Performance evaluation when non-discretionary factors correlate with technical efficiency. European Journal of Operational Research, 159, 250-257, 2004.

13. Ruggiero, J., Non-discretionary inputs in data envelopment analysis. European Journal of Operational Research 111, 461-469, 1998.

14. $\mathrm{Yu}, \mathrm{C}$, The effects of exogenous variables in efficiency measurement-A Mont Carlo study. European Journal of Operational Research 105, 569-580, 1998. 